\title{
Arbor
}

\section{Modelos evolutivos predarwinistas}

\author{
Andrés Galera
}

Arbor CLXXIII, 677 (Mayo), 1-16 pp.

El objetivo del trabajo es demostrar que el concepto de variación cronológica específica filética es un supuesto biológico ampliamente debatido por los naturalistas durante la primera mitad del siglo XIX, precedentemente a la formulación de la teoría darwinista. Como argumento de esta hipótesis analizaremos el ideario de científicos como Georges Cuvier, Étienne Geoffroy Saint-Hilaire, Heinrich-Georg Bronn, y Frédéric Gérard, comparando sus modelos evolutivos con el esquema presentado por Darwin posteriormente, 1859.

«Natural Selection is not Evolution». La frase pertenece al acervo intelectual de Ronald Fisher, abre las páginas de su libro The Genetical Theory of Natural selection ${ }^{1}$ publicado en 1930. La rotunda afirmación tiene un doble sentido:

I. La evolución es un fenómeno biológico complejo y la selección natural es uno de sus componentes. Conceptualización que, una década después, en su modern synthesis, Julian Huxley etiqueta con el epígrafe de heterogeneidad de la evolución: La selección natural "por sí sola es incapaz de extender el límite máximo de la variación, y, por tanto, incapaz por sí misma, de causar cambios evolutivos» ${ }^{2}$, en consecuencia la evolución será el producto de la mutación, la recombinación y la selección. El mismo Darwin reconoció este límite operativo, al margen del significado genético moderno, en la primera edición de On the Origin of Species: "What natural selection cannot do, is to modify the structure of one species, without giving it any advantage, for the good of another species» ${ }^{3}$. 
II. El uso indiscriminado del término selección natural como sinónimo de evolución ignorando su valor intrínseco como fenómeno biológico independiente. El simbolismo ha percutido históricamente y, habitualmente, los historiadores de la ciencia redactan el capítulo correspondiente a la evolución al dictado de esta sinonimia, identificando radicalmente el nombre de Darwin con la teoría de la evolución. Sin embargo, la ecuación Darwin = evolución es errónea por excluir otros modelos biológicos que sustentan idéntico principio teórico: el origen de los organismos actuales por la extinción de formas pretéritas y su sustitución por especies diferentes durante la cronología terrestre. Esta pluralidad ideológica significa que, lejos de manifestarse como un elemento aislado, el concepto de variación específica cronológica es un argumento debatido por los naturalistas desde principios del siglo XIX para explicar el origen de los seres vivos ${ }^{4}$; aunque de este hecho no se pude inferir la existencia de una doctrina transformista generalizada, pues aprobar que las especies han cambiado durante la cronología terrestre no obliga, como veremos a continuación, a aceptar que la sustitución se produzca por transformación o modificación de las especies existentes y halla un continuo filético entre los seres vivos desde su origen hasta hoy, aunque éste es el supuesto mayoritario. Como afirmaba su coetáneo Alphonse De Candolle, el sistema de Darwin sólo era «el más moderno, y al mismo tiempo el más ingenioso y el más completo de los sistemas fundados sobre la evolución de los seres organizados en la serie de los tiempos» ${ }^{5}$. Conozcamos otros sistemas.

\section{Las Revoluciones del Globo}

Junta cadáveres hubiera sido un apodo adecuado para el naturalista francés Georges Cuvier, aludiendo a su habilidad para recomponer esqueletos fósiles. Tarea que realizó con precisión llegando a comprender tan profundamente las relaciones anatómicas de los vertebrados que pequeñas porciones de huesos bastaban a este mago del osario, nominación que recibía en la época, para reconstruir el armazón óseo de la víctima. El descubrimiento de especies extintas inculca en Cuvier una razonable duda biogenésica bidireccional: ¿porqué han desaparecido estos organismos?, ¿qué vínculos mantiene la fauna actual con la especies desaparecidas? Como respuesta elaboró una teoría paleontológica sobre el desarrollo conjunto de la vida y la orografía terrestre, que expuso el año 1812 en el Discours préliminaire de su obra Recherches sur les ossements fossiles de quadrupèdes ${ }^{6}$. El texto tuvo éxito y su difusión internacional fue amplia, traduciéndose al inglés inmediatamente ${ }^{7}$. 
Incluyendo en nuestro análisis el ideario de Cuvier no pretendemos catalogar su teoría como un modelo evolutivo, la intención es otra y doble. Primero, liberarla del exagerado cliché catastrofista-creacionista que, generalmente, se le atribuye; segundo, demostrar que los modelos fijistas decimonónicos admiten la idea de cambio específico cronológico y rechazan el esquema transformista, que la evolución representa, defendiendo criterios científicos acordes con una coherente línea argumental y no como consecuencia de una actitud intolerante. Cuvier construye su teoría utilizando el registro estratigráfico como base empírica de su argumentación, combinando inducción y deducción para realizar su lectura. El descubrimiento de restos fósiles pertenecientes a especies desaparecidas prueba, irrefutablemente, que la fauna actual ha cambiado respecto a los antiguos moradores del mismo territorio. La causa de su desaparición se infiere también de las pruebas paleontológicas. Los fósiles conchíferos hallados en las regiones montañosas certifican la inundación sufrida por la zona continental e indican el nivel alcanzado por el agua, que es el agente físico responsable de la revolución del globo $^{8}$. La deducción alcanza cotas mayores. La presencia en los distintos estratos geológicos de restos conchíferos propios de especies muy diferentes, probaría que la involución acuática del medio no fue un fenómeno único en la historia de la Tierra sino reiterado, habría ocurrido, alternativamente, en distintas épocas: "Los cambios ocurridos en las producciones de los lechos conchíferos no han, pues, dependido solamente de una retirada gradual y general de las aguas; sino de diversas irrupciones y retiradas sucesivas, cuyo resultado definitivo ha sido, sin embargo, una disminución universal del nivel» ${ }^{9}$. Las diversas revoluciones acaecidas fueron súbitas, provocaron el aumento y posterior descenso del nivel hídrico, y la consiguiente mortandad de los organismos afectados, siendo el Diluvio Universal la última manifestación anterior a la expansión humana terrestre, pues «la vida no ha existido siempre sobre el globo, y es fácil para el observador reconocer el punto donde ella ha comenzado a depositar sus productos» ${ }^{10}$. La estructura cristalográfica y estratigráfica, y la composición material que caracterizan las elevadas cumbres de las primitivas cadenas montañosas, carentes de fósiles y fruto de la solidificación del medio fluido propio del planeta originalmente, demuestran que la aparición de los seres vivos es un proceso posterior a la génesis orográfica y la temprana inmersión de la litosfera ${ }^{11}$.

Dividida la historia de la Tierra en dos fases, inorgánica y biótica, y caracterizada su cronología biológica por los cambios faunísticos y florísticos 
acaecidos a consecuencia de las periódicas catástrofes ambientales, $\mathrm{Cu}$ vier debe, ineludiblemente, explicar ¿cuál es el mecanismo de sustitución de las especies? La respuesta es concluyente: «las especies perdidas no son variedades de las especies vivientes» ${ }^{12}$. «Esta objeción debe sobre todo parecer fuerte a quienes creen en la posibilidad indefinida de la alteración de las formas en los cuerpos organizados, y que piensan que con los siglos y las costumbres, todas las especies podrían cambiarse las unas en las otras, o resultar de una sola de entre ellas» ${ }^{13}$. Porqué las especies actuales no tienen su origen en precedentes formas modificadas. Su negativa a reconocer el hilo conductor que uniría pasado y presente no es metafísica ni respalda creencia religiosa alguna, es un argumento científico cuyo eco persiste todavía en el debate evolucionista: faltan pruebas paleontológicas de la gradación orgánica necesaria para justificar la hipótesis transformista, «si las especies han cambiado por grados, se debería encontrar trazas de estas modificaciones graduales; que entre el paleotherium y las especies de hoy se debería descubrir algunas formas intermedias, y hasta el presente eso no ha sucedido» ${ }^{14}$. Entonces ¿cómo acontece la sustitución de especies que los fósiles testimonian? La sutil respuesta compagina fijismo y variación cronológica sin abandonar el cauce científico: «no pretendo que haya sido necesaria una creación nueva para producir las especies existentes, digo sólo que ellas no existían en el mismo lugar, y que han debido venir de otra parte» ${ }^{15}$. Los territorios despoblados tras la catástrofe son colonizados por especies procedentes de otras áreas geográficas que no han sido afectadas por la involución. El supuesto migratorio es sencillo, sólo requiere limitar los efectos fenomenológicos en el espacio y en el tiempo. Temporalmente para justificar la sucesión de los cambios, la variabilidad cronológica fósil, y espacialmente para justificar empíricamente la procedencia de las nuevas especies. Utilicemos el ejemplo expuesto por Cuvier y supongamos que una gran inundación cubre la isla de Nueva Holanda. La consecuencia inmediata sería la desaparición de los organismos terrestres, entre ellos especies autóctonas, como el canguro y el ornitorrinco, que se extinguirían por su localismo permaneciendo sólo su evidencia fósil. Si el posterior descenso hídrico provocase tanto la emersión insular como la aparición de un canal terrestre entre la isla y el colindante continente asiático, este sería el camino por donde especies como el elefante, el camello, el rinoceronte, repoblarían el territorio reemplazando la precedente fauna local por animales desconocidos en dicha zona geográfica, organismos muy diferentes al contenido del registro paleontológico ${ }^{16}$. La idea admite múltiples combinaciones, necesarias para conjugar la fijeza y pluralidad de las especies que habitan el mismo territorio en distintas etapas de la cronología terrestre. 


\section{Modelos evolutivos predarwinistas}

$\mathrm{Al}$ margen de los errores que al viento de nuestro actual nivel cognoscitivo podamos señalar, la fórmula propuesta por Cuvier para explicar la historia de los seres vivos tiene el acierto de situar el problema transformista dentro de un plano puramente científico, aceptando la variabilidad específica cronológica como un hecho probado por el registro paleontológico y característico de las diferentes épocas geológicas terrestres. En adelante, la cuestión a litigar entre fijistas y transformistas no es qué ha sucedido sino cómo se producen los cambios.

\section{Las leyes paleontológicas sobre la evolución del mundo orgánico}

En 1850 la Academia de Ciencias de París convocó un concurso temático sobre el significado biológico del registro fósil ${ }^{17}$. Transcurrido el plazo oficial de tres años, la convocatoria fue declarada desierta abriéndose una nueva fase para el siguiente trienio. En la segunda ocasión, 1856, el paleontólogo alemán Heinrich-Georg Bronn ${ }^{18}$ recibe el premio por su memoria Recherches sur les lois d'évolution du monde organique pendant la formation de la croûte terrestre. Dos años después, 1858, el texto, ampliado y actualizado, se publica en alemán ${ }^{19}$, y el siguiente año aparecen sendos extractos en inglés y francés ${ }^{20}$ que difunden su ideario globalmente. Bronn había examinado más de 27.000 fósiles, una cifra récord entonces, recogidos en un voluminoso Index paleontologicus que supera las 2400 páginas ${ }^{21}$. Con esta información elaboró una teoría sobre el origen de los organismos cuya hipótesis es el cambio progresivo de la flora y la fauna por la extinción de especies existentes y la aparición de nuevas formas. El registro fósil demostraba que la historia de la vida se correspondía con un proceso biológico continuo de destrucción y génesis de nuevas especies «sin que haya habido jamás paso gradual de una especies a otra ${ }^{22}$, la relación morfológica entre las especies características de cada época era saltacionista. Bronn, igual que Cuvier, rechazaba el gradualismo fósil. Animales y plantas coinciden en su origen, manifestándose simultáneamente, y desde entonces la tipología y el número de especies cambian en el tiempo y el espacio según las características medioambientales y las posibilidades de supervivencia deri$\operatorname{vadas}^{23}$. Tres factores determinan la secuencia cronológica biogenésica: adaptación al medio, cambios geológicos, y desarrollo tipológico progre$\operatorname{sivo}^{24}$. Las propiedades del medio condicionan la proliferación de organismos produciendo una fauna y flora características de cada época ${ }^{25}$; paralelamente, la drástica transformación sufrida por la superficie te- 
rrestre sustituyendo el medio acuoso por una orografía continental, diversifica la vida multiplicando los tipos y el número de especies. El primitivo océano universal se concentra en mares, emergen los continentes y se elevan las montañosas, la pluralidad de medios hace posible la diversidad animal y vegetal, los originarios pobladores pelágicos primero se combinan con formas litorales, posteriormente se incorporan las poblaciones terrestres costeras, y finalmente las continentales. Esta serie fenomenológica «es lo que designamos bajo el nombre de evolution terripétale» ${ }^{26}$, y constituye una serie homogénea de acontecimientos: «Las primeras plantas terrestres (si no tenemos en cuenta las hullas de Portugal cuya origen siluriano es dudoso) datan de la formación devónica; los primeros animales anfibios aparecen en número considerable en la misma época. Los primeros verdaderos habitantes terrestres, que respiran el aire puro (insectos), y que caminan, aparecen en la formación carbonífera. Desde entonces, el número de los organismos terrestres ha ido siempre en aumento, y termina por superar a los organismos marinos» ${ }^{27}$. Paralelamente, el concepto de desarrollo progresivo representa la complejidad tipológica creciente del registro fósil en el decurso geológico, y define una secuencia de perfección que acontece en una doble vertiente: conduce a las nuevas especies a poseer un grado organizativo más elevado que sus antecesores y, simultáneamente, conlleva el declive de las especies pretérita una vez alcanzado el cenit hasta la desaparición total ${ }^{28}$. Pluralidad de cambios biológicos que resultan del «desarrollo del plan de creación durante la sucesión de las edades geológicas habiendo tenido lugar con una perfecta consecuencia y de una manera totalmente independiente ${ }^{29}$. Creación significa ignorancia, desconocimiento del mecanismo por el cual aparecen las nuevas especies que sustituyen a las antecesoras, y representa la secuencia de animales y plantas «que se suceden los unos a los otros sin interrupción, confundiéndose más o menos» ${ }^{30}$.

La paleontología es una disciplina básicamente ideográfica y, en consecuencia, tiene una finalidad descriptiva que Bronn no transgrede especulando sobre mecanismos evolutivos. Su obra confirma que el debate paleontológico precedente a la aparición del darwinismo se encontraba ya inmerso en la polémica transformista. Los datos demostraban el cambio orgánico pero éste no se producía siguiendo la serie gradual que la selección natural darwinista predice, por el contrario, atendiendo al registro fósil la evolución era un proceso saltacionista. A pesar de la divergencia interpretativa, Bronn contribuyó a la difusión del ideario darwinista en Alemania traduciendo On the Origin of Species en 1867, libro que había reseñado con anterioridad ${ }^{31}$. 


\section{Modelos evolutivos predarwinistas}

\section{Teratología evolutiva}

Contemplada a través del prisma embriológico, la teoría de la evolución adquiere un tinte diferente al color que le confiere la paleontología. Bajo esta nueva perspectiva sí cabe preguntarse ¿cómo se transforman las especies? La dimensión temporal de la evolución es distinta, la investigación olvida el pasado para descubrir cuál es el mecanismo biológico atemporal responsable del proceso. El cambio de perspectiva exige también un cambio metodológico pues describir ya no es suficiente, descubrir las leyes de la vida requiere del experimento. Este fue el contexto elegido por el anatomista francés Étienne Geoffroy Saint-Hilaire para elaborar una teoría sobre la evolución de los organismos sustentada en la teratología como causa general de los cambios tipológicos que manifiestan los fósiles. Planteamiento que se inserta en el marco de su teoría general sobre la unidad estructural del reino animal, «unité du plan de composition", cuya hipótesis justifica las relaciones anatómicas que determinan la existencia de un vínculo filogenético entre las distintas tipologías a través del concepto de analogía orgánica ${ }^{32}$.

La existencia de una correlación entre desarrollo embrionario y evolutivo fue una suposición temprana en la biología del siglo XIX. La idea de recapitulación aparece en diferentes versiones durante las primeras décadas del siglo, la suscriben autores como Treviranus, Serres, Oken, Tiedemamm, Merck. Los embriones de las clases superiores repiten en su desarrollo las formas permanentes de las clases inferiores, afirma, por ejemplo, Serres en su Anatomía comparada del cerebro ${ }^{33}$. En este sentido, la teoría evolutiva de Saint-Hilaire significa novedad pues, además, considera el desarrollo embrionario como la fase operativa donde acontecen los cambios organográficos responsables de la aparición de nuevos fenotipos. El paleontólogo Etienne deduce fácilmente que «los animales que viven hoy en día proceden, por una secuencia de generación ininterrumpida, de animales perdidos del mundo antediluviano» ${ }^{34}$; argumento que el anatomista Geoffroy refrenda siguiendo el principio de unidad anatómica de los seres vivos; y la finalidad del embriólogo Saint-Hilaire es determinar las causas del cambio morfológico. Como afirma su contemporáneo Quatrefages, su objetivo era resolver una de las objeciones más importantes que la teoría evolutiva puede recibir: «la dificultad de comprender como dos especies, hasta entonces reunidas fisiológicamente, se aíslan» ${ }^{35}$. La teratología era la solución. Animales regulares y monstruos representan sólo diferentes grados de organización de la materia viva, ocurrida durante la organogénesi ${ }^{36}$, y su coexistencia es una prueba empírica tanto del potencial transformista de los seres vivos como 
de la posibilidad real de que una especie sea sustituida por otra morfológicamente distinta vinculada genésicamente.

Aceptado el esquema teratológico como motor de la evolución es necesario indagar ¿cuál es la causa de las alteraciones embrionarias? Entendida la historia de la vida como lo hace Saint-Hilaire: los objetos naturales una vez formados por la deidad «son definitivamente abandonados a su propia aptitud $»^{37}$, se deduce que el origen de la vida fue un acto creacionista y que los primigenios seres vivos se desarrollan posteriormente mediante un proceso evolutivo cuya causa es inherente a su entidad biológica y a su relación con el medio. Dado su carácter presentista la hipótesis requiere la comprobación experimental. Su investigación se centró en estudiar la embriogénesis de las aves, y consistía en observar el desarrollo del huevo sometido a distintas modificaciones durante las diferentes etapas de la incubación: agitando, perforando, manteniendo en posición vertical, impermeabilizando la cascara, etc. Los polluelos presentaban numerosas alteraciones organográficas, monstruosidades, y como las anomalías no estaban predeterminadas en el huevo, que en circunstancias normales no se modifica, los cambios eran «resultado de una perturbación, ocurrida en el curso del desarrollo de los embriones en principio perfectamente regulares» ${ }^{38}$. La lectura de los resultados en clave evolutiva permite afirmar que, por influencia del medio y a causa de los cambios morfológicos derivados de esta relación, las especies animales y vegetales son reemplazadas por otras que continúan «a su vez todo este lecho de organización animal y vegetal de que está compuesta la corteza del globo terrestre» ${ }^{39}$. La naturaleza embriológica del problema sitúa la evolución en un plano puramente genésico delimitado por dos factores: los caracteres morfológicos contenidos en el huevo y el mundo exterior ${ }^{40}$. El germen se diferencia en un individuo adulto adaptado a un determinado ambiente, nisus formativus, cuando el nicho formativo se modifica los cambios afectan al desarrollo de los futuros embriones modificando la organogénesis y originando nuevas morfologías; por ejemplo, «la cantidad decreciente de oxígeno en relación con los demás componentes atmosféricos, pudo forzar las superficies cutáneas del embrión, primer y principal sede de los actos respiratorios, a abrirse más, a ganar en razón inversa al volumen existente de oxígeno, más profundidad, mediante más largas sinuosidades en el tejido celular, y adquirir, por un crecimiento en la intensidad de los efectos, de más en más, el carácter de burbujas y decididamente de traqueas, hasta que por fin aparecen en el tórax una concentración de senos respiratorios, y de los arreglos de la estructura para el aislamiento de los sacos o escenarios de la respiración, llamados, según sus características, pulmones o branquias» ${ }^{41}$. Las nue- 
vas características morfológicas son adaptativas y responden a las condiciones de existencia determinadas por los cambios del medio, se habrá constituido un nuevo nisus formativo. El resultado del proceso es la extinción de las especies parentales, ahora inadaptadas, que son sustituidas por la descendencia modificada.

La teratología evolutiva de Saint-Hilaire analiza la evolución desde el punto de vista de la reproducción de los seres vivos, definiéndola como un fenómeno de adaptación de nuevas formas a nuevos medios mediante un limitado mecanismo selectivo, entre las formas parentales y su descendencia, controlado y dirigido por el medio (selección no competitiva), y en su horizonte se vislumbra el hopeful monster ${ }^{42}$ propuesto por Richard Goldschmidt en el siglo XX, ahora bajo los auspicios de la genética en armoniosa conjunción embriológica.

\section{La teoría de la evolución de las formas orgánicas}

Es sabido que el empleo del término evolución con su actual perfil biológico no es una aportación darwinista, su uso se remonta a la escuela naturalista francesa de principios del siglo $\mathrm{XX}^{43}$. Tampoco fue original la formulación de una teoría sobre el origen de las especies mediante la sustitución y extinción de las formas parentales por nuevas especies vinculadas genealógicamente; al margen de Lamarck, cuyo ideario implica la transformación morfológica directa del individuo en otro con cualidades anatómicas diferentes, por adaptación al medio, y la consiguiente transmisión a su descendencia de los caracteres adquiridos. Esquema admitido también por Darwin. Durante la década de los años cuarenta el Diccionario Universal de Historia Natural, dirigido por Charles d'Orbigny, fue la tribuna pública donde otro naturalista francés, Frédéric Gérard, expone la théorie de l'évolution des formes organiques ${ }^{44}$. Su objetivo era explicar cómo surgió la vida sobre la Tierra y determinar cuál fue el desarrollo posterior de los seres vivos hasta alcanzar la actual diversidad de especies ${ }^{45}$. Consecuente con su interpretación fisicalista de la naturaleza -las leyes físicas rigen los fenómenos naturales, incluida la evolución $^{46}$-, la vida es un estado de la materia ${ }^{47}$ y su primigenia manifestación ocurre por generación espontanea durante la fase anorgánica terrestre ${ }^{48}$. La vida surge mediante la acción de agentes físicos, "calor, luz, electricidad y nada más» ${ }^{49}$, sobre «un líquido proveniente sea del agua pluvial, sea de una infusión ${ }^{50}$. Desde entonces, los seres vivos se habrían desarrollado en múltiples direcciones morfológicas sucediéndose las distintas especies en el espacio y el tiempo por un proceso de trans- 
formación que implica la extinción de las formas existentes y su sustitución por otras diferentes adaptadas a las nuevas condiciones de existencia impuestas por los cambios del medio: «las condiciones de existencia propias a la aparición de seres de tal o cual orden no han existido simultáneamente, y que las evoluciones sucesivas no son otras que las formas orgánicas correspondientes a las circunstancias ambientales» ${ }^{51}$. La imagen de este sistema evolutivo corresponde a una secuencia continua de organismos de complejidad ascendente ${ }^{52}$, que van desde las formas simples de los primeros tiempos hasta la diversidad actual, fruto del incesante juego de variabilidad fenotípica que caracteriza a la naturaleza y hace de la evolución una ley biológica general ${ }^{53}$.

El soporte empírico de la teoría es la consabida deducción paleontológica: la relación estratigráfica de fósiles pertenecientes a organismos desaparecidos prueba la sucesión de especies animales y vegetales ocurrida durante la historia terrestre, ¿cómo explicar sino la «presencia de restos orgánicos sepultados en el seno de los lechos de las diferentes épocas, y que nos han obligado a crear tantos nombres nuevos, ante la imposibilidad de relacionarlos con los tipos que existen actualmente?»54. Junto al testimonio del pasado, la morfogénesis, la embriología, retrata hoy la historia de la vida -teoría de la recapitulación-, confirmando la «idea de la evolución sucesiva de los seres desde los más simples hasta los más complejos, de la que cada grupo, cada especie, representa uno de los puntos de la evolución ${ }^{55}$. Establecida la relación filogenética subyacente al registro paleontológico, definida como un proceso continuo de transformaciones orgánicas, aceptar la teoría de la evolución implica aceptar un principio de unidad anatómica, extensivo a todos los seres vivos, que dé verosimilitud al cambio morfológico y haga posible la idea de continuidad orgánica. Gérard sustenta su hipótesis transformista en el modelo de unidad de composición anatómica defendido por su correligionario Etienne Saint-Hilaire: los seres vivos representan un plan estructural único que ha sufrido modificaciones sucesivas relacionadas con las diferentes condiciones de existencia que cronológicamente han caracterizado el plane$t^{56}$. Pero aún quedan interrogantes por responder: ¿cuál es el mecanismo biológico responsable de los cambios orgánicos?, ¿dónde están los fósiles intermedios que den concordancia al continuo filogenético, como preguntaba Cuvier? La respuesta es común, no hay relación causa-efecto entre los diferentes componentes del problema. La prueba paleontológica de la evolución no es la frecuencia de sustitución sino el acto del cambio específico que testimonian los fósiles. La paleontología demuestra la evolución de las especies independientemente de nuestra ignorancia sobre los mecanismos biológicos y las lagunas presentes en el registro fósil: «El 
proceso de transformación, la manera cómo estos cambios se han podido operar, no es conocida, y no se sabe el tiempo que será necesario para ello», lo cierto es "que ha habido un movimiento evolutivo» ${ }^{57}$.

En síntesis, la théorie de l'évolution des formes organiques se desglosa en tres vertientes conceptuales: el origen de la vida, la transformación orgánica, y el orden de sucesión de las especies ${ }^{58}$. A partir de una imaginaria primigenia forma viva, utópica célula primitiva ${ }^{59}$, la vida se desdobla en dos niveles organizativos, animales y vegetales, sustrato donde, resultado de la evolución, se diferenciaran los distintos tipos morfológicos conocidos: radiolarios, moluscos, articulados, peces, reptiles, pájaros, mamíferos, acotiledóneas, monocotiledóneas y dicotiledóneas ${ }^{60}$, representados por un conglomerado de especies que conforman la historia biológica de la Tierra dividida en siete períodos evolutivos (anorgánico y orgánico primordial, carbonífero, jurásico, cretácico, terciario, aluvial y moderno $)^{61}$, caracterizados por una fauna y flora peculiares acordes a las condiciones del medio y las posibilidades de existencia derivadas. Los diferentes tipos se mantienen constantes desde su aparición hasta la actualidad a través de representantes diferentes que reemplazan a sus antecesores, actuando la ley de la evolución en dos direcciones ${ }^{62}$ : sustitución de unas especies por otras dentro de un tipo establecido (fenotípicamente correspondería a simples modificaciones orgánicas); aparición de nuevos tipos (alteraciones morfológicas drásticas dentro de la unidad estructural original). Planteamiento dual que, implícitamente, sitúa la teoría de la evolución de Gerard en el marco de uno de los problemas capitales del futuro debate evolucionista: la macro y la microevolución. En ambos casos los cambios incrementan la complejidad anatómica, su finalidad es adaptativa y tienen un significado binario implícito: supervivencia y expansión territorial. La relación del ser vivo con el medio está determinada por las condiciones de existencia que los cambios geológicos establecen, las modificaciones orgánicas son la respuesta adaptativa a la nueva situación ambiental, son necesarias para sobrevivir. Paralelamente, la historia geológica terrestre discurre en la dirección que marca la emergencia continental del medio acuático originando nuevos hábitats (aire, tierra) factibles de colonizar ${ }^{63}$. La respuesta morfológica supone ahora un cambio fenotípico radical también regulado por el binomio adaptaciónsupervivencia pero dirigido a colonizar nuevos territorios. En este juego de variaciones ¿cuál es el significado del concepto de especie? se pregunta Gérard siguiendo el hilo conductor de la teoría. Bajo el prisma de la evolución, aceptado su valor temporal, las especies representarían unidades tipológicas arbitrarias, relativas, son estados transitorios de la vida «correspondientes a los diferentes peldaños de la evolución de los 
cuerpos vivos» ${ }^{64}$. La especie es un concepto que identifica agrupaciones de individuos con identidad formal, organizativa, costumbrista, reproductora, temporalmente coincidentes e inestables por la movilidad morfológica que razas, variedades, e híbridos representan ${ }^{65}$ como manifestación primera del continuo juego de cambios biológicos resultado de la ley de la evolución. Frédéric Gérard interpreta la historia de la vida haciendo una lectura continuada del registro fósil que aúna pasado y presente mediante una secuencia filogenética. La extinción de especies y su sustitución por nuevas formas surgidas de las precedentes es un fenómeno general que caracteriza una naturaleza gobernada por agentes físicos exclusivamente.

De haber leído las páginas escritas por estos y otros científicos, Darwin habría podido seguir menospreciando el nombre de Lamarck, a pesar de confesar que «the conclusions I am led to are not widely different from his; though the means of change are wholly so» ${ }^{66}$, pero su lectura hubiera ayudado a contrarrestar su egolatría y despejar las dudas que sobre la mutabilidad de las especies todavía albergaba en la década de los años cuarenta, cuando redactaba los primeros borradores de su teoría. Y si los biólogos aprendiésemos a conocer, valorar, y emplear el acervo cognoscitivo de nuestros predecesores, serían inviables falsedades como, por ejemplo, las enunciadas por Richard Dawkins invocando a Darwin en vano, como único artífice de la teoría evolutiva ${ }^{67}$. Nuestro objetivo ha sido muy diferente, demostrar que la historia de la evolución no ocurrió tal y como la cuentan los profetas darwinistas del siglo XX.

\section{Notas}

1 Fisher, Ronald A. (1958):The Genetical Theory of Natural Selection, Dover, Nueva York, preface, p.vii ( $1^{\mathrm{a}}$ ed. 1930, Oxford University Press).

2 Huxley, J. (1965): La evolución.Síntesis moderna, Losada, Buenos Aires, $2^{\mathrm{a}}$ ed., p.29 (Evolution. The Modern Synthesis, G. Allen \& Unwin Ltd., Londres, 1943).

3 DARwin, Ch. (1859): On the Origin of Species by Means of Natural Selection, JoHN MuRRAY, Londres, p.87 (citamos por la edición facsímil realizada en 1998, Harvard University Press, Cambridge-Massachusetts-Londres).

4 Desde la tercera edición, 1861, de On the Origin of Species Darwin incorporá un bosquejo histórico sobre los antecedetes de su teoría, citando las investigaciones de Lamarck, E. G. Saint-Hilaire, W. C. Wells, W. Herbert, Robert E. Grant, P. Matthew, Omalius d'Hollay, H. Spencer, Charles Naudin, etc., como ejemplos del debate transformista-evolucionista que precedió a su obra.

5 CANDOlle, A. de (1862): «Etude sur l'espèce à l'occasion d'une révision de la famille des cupulifères", Annales des sciences naturelles, $4^{\mathrm{a}}$ serie, Botanique, t.XVIII, pp. 59110 , p.101. 
${ }^{6}$ CUVIER, G. (1812): Recherches sur les ossements fossiles de quadrupèdes, ou l'on rétablit les caractères de plusieurs espèces d'animaux que les révolutions du globe paroissent avoir détruites, París, 4 t. En el periodo 1821-1824 se publicó una edición revisada y ampliada a 5 tomos, y en 1825 se edita independiente el discurso preliminar con el título de Discours sur les révolutions de la surface du globe et sur les changements qu'elles ont produits dans le règne animal.

7 Cuvier, G. (1813): Essay on the Theory of the Earth (traducido por Robert Kerr), William Blackwood, Edimburgo ( $2^{\mathrm{a}}$ ed. 1815).

8 Cuvier, G. (1812): Discours preliminaire. Citamos por la ed. de 1992, Flammarion, París, pp.49-52.

9 Ibídem, p.54. También pp.52-53.

10 Ibídem, p.55. Sobre el hombre véase p. 122.

11 Ibídem, p.56-59.

12 Ibídem, p.111.

13 Ibídem, p.112.

14 Ibídem.

15 Ibídem, p.119

16 Ibídem, pp.119-120.

17 Véase BeAumont, L. Élie de (1850): «Question proposée pour le Grand Prix des Sciences Physiques", Compte Rendu de l'Academie des Sciences, 30, 257-260. También Laurent, G. (1997): «Paléontologie et évolution: état de la question en 1850 d'après l'oeuvre de Heinrich-Georg Bronn (1800-1862)", en De la géologie à son histoire, CTHS, París, 175-188.

18 HEINRICH-GEORG BRONN (1800-1862), fue profesor de historia natural y director de las colecciones geológicas y zoológicas de la Universidad de Heilderberg.

19 BRoNN, H.G. (1858):Untersuchungen über die Entwickelungs-Gesetze der organischen Welt während der Bildungs-Zeit unserer Erd-Oberfläche, E. Schweizerbart, Stuttgart.

20 BronN, H.G. (1859): "On the Laws of Evolution of the Organic World during the formation of the Crus of the Earth", Ann. Mag. Nat. Hist., 3, 4, (1859): 81-90, 175-184; "Recherches sur les lois d'évolution du monde organique pendant la formation de la croûte terrestre", Archives des Sciences Physiques et Naturelles, 4, (1859): 217-241. En 1861 la Académie des Sciences publicó el texto íntegro en francés, Supplément aux Compte Rendu, 2, (1861): 377-918.

21 BRonN, H.G. (1848-9): Index paleontologicus oder übersicht der bis jetzt bekannten fossilen organismen, E. Schweizerbart, Stuttgart, 3 vols.

22 BRonN, H.G. (1859): «Recherches sur les lois d'évolution du monde organique pendant la formation de la croûte terrestre", Archives des Sciences Physiques et Naturelles, 4, p.219.

23 Ibídem, p.218.

24 Ibídem, p.226.

25 Ibídem, pp.220-1.

26 Ibídem, p.222.

27 Ibídem, pp.222-3.

28 Ibídem, p.225.

29 Ibídem, p.223.

30 BronN, H. G. (1861), «Essai d'une réponse à la question de prix propose en 1850 par l'Académie des Sciences", Supplément aux Compte Rendu, 2, (1861): 377-918, p. 733. Reproducido en Laurent, G. (1997), p.181 
31 DARwin, Ch. (1867): Ueber die Entstehung der Arten durch natuerliche Zuchtwahl oder die Erhaltung der beguenstigten Rassen im Kampfe um's Dasein (aus dem engl. uebers. von H. G. Bronn. Nach der 4. engl. sehr verm. Ausg. durchges. und berichtigt von J. Victor CARUS), E. Schweizerbart, STUTTGaRT. La reseña se publicó en Neues Jahrbuch für Mineralogie, Geognosie, Geologie und Petrefakten-Kunde, 1861, pp.112-116.

32 Órganos análogos son los que tiene idéntica situación corporal y la misma composición material, modificándose de múltiples formas en las distintas especies zoológicas según la finalidad. R.Owen redefinió posteriormente el concepto denominando analogía a la semejanza funcional y homología a la semejanza anatómica, significados válidos en la actual anatomía comparada.

33 Serres, A. (1824-6): Anatomie comparée du cerveau, Gabon, París-Montpellier, 2 t.

34 Geoffroy Saint-Hilaire, E. (1831): Recherches sur de grands Sauriens trouvés à l'ètat fossile, Didor, París, p. 74; cit. en Laurent, G. (1987): Paléomtologie et évolution en France de 1800 a 1860. Une histoire des idées de Cuvier et Lamarck à Darwin, CTHS, París, p. 339.

35 Quatrefages, A. de (1870): Charles Darwin et ses précurseurs français, Baillière, París, p. 64.

36 Cf. Laurent, G. (1987), p. 337.

37 Geoffroy Saint-Hilaire, E. (1829): Cours de l'Histoire Naturelle des Mammifêres, Pichon et Didier, París, lección 18, p. 5; cit. en Laurent, G. (1987), p. 337

38 Geoffroy SAINT-HilaIre, I. (1847): Vie, travaux et doctrine scientifique de Etienne Geoffroy Daint-Hilaire, Bertrand, París, p.291.

39 Geoffroy Saint-Hilaire, E. (1831), p.41; cit. en Laurent, G. (1987), p. 340.

40 Ibídem, p. 69; también LAURENT, G. (1987), p. 344.

41 Citado en Laurent, G. (1987), p. 348.

42 GoldschmidT, R. (1933): "Some aspects of evolution", Science, 78, pp. 539-547.

43 Cf. Laurent, G. (1990): "Transformisme», Encyclopèdie Philosophique Universelle, PUF, París, vol. 2 (Les notions philosophiques. Dictionnaire); GALERA, A. (2000): "Historias de la evolución". Asclepio, pp. 1-2.

${ }^{44}$ Cf. los artículos escritos por Frédéric Gérard para el Dictionnaire Universel d'Histoire Naturelle (París, 1841-9, 13 tomos): «Géographie zoologique», 1845, t.6, pp.112-192; "Generation spontaneée ou primitive», 1845, t. 6, pp. 53-71; "Dégénérescence», 1844, t.4, pp. 647-655; «Espèce», 1844, t.5, pp. 428-452 (citamos por la $2^{\text {a }}$ edición, Abel Pilon, París 1867-1872, t.6, pp.359-455; t.7, pp.149-167; t.5, pp. 8-6; t.5, pp 659-683; respectivamente).

45 "Géographie zoologique", p. 359.

46 «Espèce", p. 670; «Géographie zoologique», p. 359.

47 «Géographie zoologique», pp. 363, 364; también «Generation spontaneée ou primitive", p. 166.

48 "Géographie zoologique», p. 368.

49 «Generation spontaneée ou primitive», p. 166.

50 "Géographie zoologique», p. 363.

51 Ibídem, p. 364.

52 Ibídem, pp. 364, 365 .

53 Ibídem, pp. 364, 366.

54 "Dégénérescence», p.10; $c f$. también «Espèce», p. 664.

55 «Espèce», p. 662; $c f$. también "Géographie zoologique», pp. 365, 367.

${ }^{56}$ Cf. "Dégénérescence», p.10; «Espèce», p.663; "Géographie zoologique», pp. 364, 366. 


\section{Modelos evolutivos predarwinistas}

57 «Espèce», p. 664-5; $c f$. también «Géographie zoologique», p. 367.

$58 C f$. «Géographie zoologique», pp. 361, 363.

59 Ibídem, p.364.

60 Ibídem.

61 Ibídem, p.367.

62 Ibídem, p.377.

63 Ibídem, pp.369 y ss.

64 "Espèce», p.683.

65 Ibídem, p.670, 682.

66 Carta de Charles Darwin a Joseph Hooker, 11 de enero de 1844. $C f$. Darwin, F. (ed.) (1958): The Autobiography of Charles Darwin and Selected letters, Dover, Nueva York ( $1^{\text {a }}$ ed. 1892$)$, p. 184.

67 Dawkins, R. (1979), The selfish gene, Oxford University Press, Nueva York.

\section{Bibliografía}

BronN, H. G. (1848-9): Index paleontologicus oder übersicht der bis jetzt bekannten Fossilen organismen, E. Schweizerbart, Stuttgart, 3 vols.

BRonN, H. G. (1858):Untersuchungen über die Entwickelungs-Gesetze der organischen Welt während der Bildungs-Zeit unserer Erd-Oberfläche, E. Schweizerbart, Stuttgart.

BronN, H. G. (1859): «On the Laws of Evolution of the Organic World during the formation of the Crus of the Earth", Ann. Mag. Nat. Hist., 3, 4, pp. 81-90, 175-184;

BRonN, H. G. (1859) «Recherches sur les lois d'évolution du monde organique pendant la formation de la croûte terrestre", Archives des Sciences Physiques et Naturelles, 4, pp. 217-241.

BronN, H. G. (1861), «Essai d'une réponse à la question de prix propose en 1850 par l'Académie des Sciences", Supplément aux Compte Rendu, 2, (1861): 377-918.

CANDOlle, A. de (1862): «Etude sur l'espèce à l'occasion d'une révision de la famille des cupulifères", Annales des sciences naturelles, $4^{\mathrm{a}}$ serie, Botanique, t.XVIII, pp. 59-110.

CUVIER, G. (1812): Recherches sur les ossements fossiles de quadrupèdes, ou l'on rétablit les caractères de plusieurs espèces d'animaux que les révolutions du globe paroissent avoir détruites, París, $4 \mathrm{t}$.

Cuvier, G. (1813): Essay on the Theory of the Earth (traducido por Robert Kerr), William Blackwood, Edimburgo ( $2 .^{\mathrm{a}}$ ed. 1815).

Cuvier, G. (1992): Discours preliminaire, Flammarion, París.

DARwiN, Ch. (1859): On the Origin of Species by Means of Natural Selection, John Murray, Londres (edición facsímil Harvard University Press, Cambridge-Massachusetts-Londres, 1998).

Fisher, Ronald A. (1958): The Genetical Theory of Natural Selection, Dover, Nueva York ( $1^{a}$ ed. 1930, Oxford University Press).

Galera, A. (comp.) (2000): "Historias de la evolución", Asclepio, CSIC, Madrid.

GÉRARD, F. (1841-9) "Géographie zoologique", "Generation spontaneée ou primitive», "Dégénérescence», "Espèce», Dictionnaire Universel d'Histoire Naturelle, París, 13 t.

Geoffroy SaInT-Hilaire, E. (1831): Recherches sur de grands Sauriens trouvés à l'ètat fossile, Didor, París. 


\section{Andrés Galera}

Geoffroy SaINT-Hilaire, E. (1829): Cours de l'Histoire Naturelle des Mammiferes, Pichon et Didier, París.

Geoffroy SAINT-HILAIRE, I. (1847): Vie, travaux et doctrine scientifique de Etienne Geoffroy Saint-Hilaire, Bertrand, París.

GolDSCHMIDT, R. (1933): «Some aspects of evolution", Science, 78, pp. 539-547.

GoldschmidT, R. (1940): The Material Basis of Evolution, Yale University Press, New Haven.

HuXley, J. (1965): La evolución.Síntesis moderna, Losada, Buenos Aires, $2^{\mathrm{a}}$ ed. (Evolution. The Modern Synthesis, G. Allen \& Unwin Ltd., Londres, 1943).

Laurent, G. (1987): Paléomtologie et évolution en France de 1800 a 1860. Une histoire des idées de Cuvier et Lamarck à Darwin, CTHS, París.

LAURENT, G. (1990): “Transformisme», Encyclopèdie Philosophique Universelle, PUF, París, vol. 2 (Les notions philosophiques. Dictionnaire).

LAURENT, G. (1997): «Paléontologie et evolution: état de la question en 1850 d'après l'oeuvre de Heinrich-Georg Bronn (1800-1862)", De la géologie à son histoire, CTHS, París, pp. 175-188.

QuATrefages, A. de (1870): Charles Darwin et ses précurseurs français, Baillière, París. SERres, A. (1824-6): Anatomie comparée du cerveau, Gabon, París-Montpellier, 2 t. 